\title{
Modelo de toma de decisiones bioéticas en ciencias de la salud
}

\author{
Nancy Piedad Molina Montoya
}

\begin{abstract}
Resumen: La importancia de la toma de decisiones éticas en ciencias de la salud ha promovido la generación de esquemas que orientan este proceso. El objetivo es proponer un modelo teórico de toma de decisiones bioéticas. Se hizo una búsqueda y revisión de la literatura en bases de datos y bibliotecas electrónicas con palabras clave, se identificaron y analizaron once modelos de toma de decisiones éticas según parámetros establecidos y se incluyeron resultados de estudios sobre el tema. Los esquemas analizados plantean una toma de decisiones basada en la razón a partir de un número variable de pasos cognitivos y, en general, aportan pocos elementos para su aplicación práctica. Se planteó el Modelo de Integración Razón Emoción para la Toma de Decisiones Bioéticas en Ciencias de la Salud. Este modelo sintetiza los pasos cognitivos para tomar decisiones adecuadas, mediante la integración de la razón y la emoción, la posibilidad de aplicación de diversos marcos éticos y la puesta en juego de la competencia ética y las habilidades de pensamiento crítico.
\end{abstract}

Palabras clave: bioética; educación; moral; salud; toma de decisiones

Fecha de Recepción: 06 de agosto de 2018

Fecha de aceptación: 26 de novimebre de 2018
Fecha de Evaluación: 14 de septiembre de 2018

Publicación en Línea: 19 de diciembre de 2018

Cómo citar: Molina Montoya, N. P. (2019). Modelo de Toma De Decisiones Bioéticas En Ciencias de la Salud. Revista Latinoamericana de Bioética, 19(36-1). https://doi.org/10.18359/rlbi.3598

a Optómetra, Msc. Ph.D. Docente investigadora de la Universidad de La Salle. Bogotá, Colombia.

Correo electrónico: nanmolina@unisalle.edu.co

ORCID: https://orcid.org/0000-0001-5239-1304 


\section{Model of Bioethical Decision Making in Health Sciences}

Abstract: The importance of ethical decision making in health sciences has promoted the generation of schemes that guide this process. The objective is to propose a theoretical model of bioethical decision making. We searched and reviewed the literature in databases and electronic libraries with keywords, eleven models of ethical decision making were identified and analyzed according to established parameters and results of studies on the subject were included. The schemes analyzed propose a decision making process based on a variable number of cognitive steps and, in general, provide few elements for their practical application. The Reason-Emotion Integration Model for Bioethical Decision Making in Health Sciences was proposed. This model synthesizes the cognitive steps to make appropriate decisions, by integrating reason and emotion, the possibility of applying various ethical frameworks and implementing an ethical competence and critical thinking skills.

Key words: bioethics; education; moral; health; decision making

\section{Modelo de tomada de decisões bioéticas em ciências da saúde}

Resumo: Aimportância da tomada de decisões éticas em ciências da saúde tem promovido a geração de esquemas que orientam esse processo. O objetivo é propor um modelo teórico de tomada de decisões bioéticas. Fez-se uma busca e revisão da literatura em bancos de dados e bibliotecas eletrônicas com palavras-chave, identificaram-se e analisaram onze modelos de tomada de decisões éticas segundo parâmetros estabelecidos e incluíram-se resultados de estudos sobre o tema. Os esquemas analisados propõem uma tomada de decisões baseada na razão a partir de um número variável de passos cognitivos e, em geral, contribuem com poucos elementos para sua aplicação prática. Propôs-se o Modelo de Integração Razão Emoção para a Tomada de Decisões Bioéticas em Ciências da Saúde. Esse modelo sintetiza os passos cognitivos para tomar decisões adequadas, mediante a integração da razão e da emoção, a possibilidade de aplicação de diversas estruturas éticas e a implementação da competência ética e as habilidades de pensamento crítico.

Palavras chave: bioética; educação; moral; saúde; tomada de decisões 


\section{Introducción}

Cerit y Dinc afirman que una decisión clínica tiene un componente ético si está basada en un juicio de valor sobre las consecuencias de la decisión o si evalúa el cumplimiento de los deberes y derechos de los implicados (Cerit y Dinc, 2012). Diferentes estudios (House, Theyyunni, Barnosky, Fuhrel-Forbis, Seeyave, Dawn y Santen, 2015; Molina, 2016; Sinclair, 2013 y Shapiro y Miller, 1994) reportan que los profesionales y estudiantes en ciencias de la salud deben tomar a diario decisiones éticas en su práctica clínica como respuesta a dilemas o problemas morales de diversa naturaleza en relación con la labor que desempeñan.

La toma de decisiones éticas es un proceso compuesto por diferentes fases que incluyen la identificación de un dilema o problema, la búsqueda de información para ampliar su comprensión, el análisis y el planteamiento de alternativas de solución, el balanceo de estas alternativas y la elección de una opción moralmente justificada que lleva a tomar la decisión. En las últimas décadas, desde el campo de la bioética ha crecido el interés en el desarrollo de guías orientadoras que buscan proporcionar respuestas más consistentes, profundas, soportadas y moralmente aceptadas (Kader, 2005). Esta es la razón por la que se encuentran en la literatura diversos modelos de toma de decisiones éticas.

Los modelos de toma de decisiones son esquemas teóricos que se constituye en puntos de referencia. Según Grundstein-Amado, estos funcionan como dispositivos intelectuales que simplifican y clarifican las fuentes de perplejidad moral, permitiendo a cada uno llegar a una elección autodirigida a través del seguimiento de una lista variable de pasos cognitivos (Grundstein-Amado, 1991). En contextos de salud, orientan a los profesionales para resolver los asuntos clínicos que se presentan en los servicios de salud.

En efecto, investigaciones con estudiantes de enfermería (Cameron et al., 2000 y Park, Cameron, Han, Ahn, Oh y Kim, 2003) muestran que la utilización de un modelo de toma de decisiones para resolver los problemas éticos en la práctica de enfermería fue considerada útil.
La importancia de toma de decisiones éticas en ciencias de la salud requiere de la promoción de esta habilidad en la formación bioética de estudiantes y profesionales, para lo cual se precisa de la planeación cuidadosa de los contenidos y la elección de estrategias de enseñanza aprendizaje en coherencia con las expectativas y necesidades de los profesionales en formación.

Es ideal, entonces, contar con modelos de toma de decisiones éticas que aporten elementos para su aplicación práctica en diferentes ámbitos. Por esta razón, el propósito del presente artículo es proponer un modelo de toma de decisiones bioéticas, con base en la revisión de la literatura.

\section{Materiales y métodos}

Este es un artículo de revisión documental. Se realizó una búsqueda de artículos en las bases de datos ebsco, Proquest, en la biblioteca científica Scielo y en Google Académico con base en los términos $\mathrm{MeSH}$ (medical subjects headings) ética, bioética y toma de decisiones, con los que se construyeron ecuaciones de búsqueda incorporando palabras como modelo, marco o proceso en inglés y español. No se limitó el periodo de búsqueda. Se tuvieron en cuenta los artículos o documentos con base en el objetivo propuesto. Se leyó cada artículo, se identificó cada modelo, el supuesto teórico de base, los pasos cognitivos para la toma de decisiones y el planteamiento de pautas para su aplicación. Se transfirieron datos a una matriz, con el fin de hacer un ejercicio comparativo de identificación y análisis de los más importantes para la toma de decisiones bioéticas. Adicionalmente, se analizaron los resultados de la investigación sobre toma de decisiones éticas para complementar la construcción del modelo.

La primera búsqueda se realizó en el año 2015 como parte del proyecto de tesis doctoral de la autora. Los resultados de esta investigación fueron tenidos en cuenta para el planteamiento del modelo. En diciembre de 2017 se actualizó la búsqueda siguiendo los mismos criterios que en la primera revisión, con el fin de revisar nuevos artículos que pudieran haberse publicado recientemente para incorporarlos al planteamiento del modelo. 


\section{Resultados}

\section{Modelo de toma de decisiones éticas}

A partir de la búsqueda se identificaron 15 modelos de toma de decisiones éticas. Se excluyeron 4 modelos (Cassells, Jenkins, Lea, Calzone y Johnson, 2003; Schmidt, 2008; Toren, 2010; Wueste, 2005), debido a que no presentaban los supuestos teóricos en los que están basados o la lista de pasos cognitivos para alcanzar la toma de decisiones. El análisis se hizo sobre los 11 modelos restantes. En la tabla 1 se hace una breve reseña de cada uno.
A partir de la revisión, se pudo identificar que el proceso de toma de decisiones éticas incluye varios pasos cognitivos y componentes que conllevan el juicio moral y la justificación de la decisión (Cerit y Dinc, 2012). Estos pueden sintetizarse en cinco fases: 1) análisis de la información y reconocimiento del problema, 2) identificación de los cursos de acción y exploración de los posibles resultados, 3) elección de una opción y justificación moral de la misma, 4) implementación de la acción, y 5) monitoreo y seguimiento de la decisión. Los pasos cognitivos de cada modelo y la comparación entre ellos se muestran en la tabla 2.

Tabla 1. Modelos de Toma de decisiones éticas

\begin{tabular}{l|l|l}
\hline$N^{\circ}$ & \multicolumn{1}{c|}{ Autor (es) } & \multicolumn{1}{c}{ Descripción } \\
\hline 1 Thompson, J. (1985) & $\begin{array}{l}\text { Es un modelo de } 10 \text { pasos para la toma de decisiones bioéticas que utiliza la investigación crítica y el } \\
\text { razonamiento moral. }\end{array}$ \\
\hline $\begin{array}{l}\text { Grundstein-Amado, } \\
\text { R. (1991) }\end{array}$ & $\begin{array}{l}\text { El modelo está compuesto por tres partes: el marco ético: determinado por la estructura del } \\
\text { razonamiento moral del individuo y está basado en el sistema de valores del individuo (morales y } \\
\text { el curso final de la acción (basada en la teoría clásica de toma de decisiones) y el marco contextual: } \\
\text { aspectos relacionales entre el paciente y el profesional y el impacto de la estructura organizacional en } \\
\text { el comportamiento ético del mismo. }\end{array}$ \\
\hline
\end{tabular}

Fue diseñado y desarrollado para ilustrar una concepción general de la interacción entre la enfermera y el cliente dentro de un marco ético. Este modelo se presta al examen de todos los factores que entran en el proceso de toma de decisiones: descriptivo, normativo y meta ética. La teoría subyacente es la

3 Greipp, M.E. (1992) teoría general de sistemas. Este modelo es compatible con el trabajo extensivo realizado por Leininger y su teoría de cuidado transcultural (Leininger, 1978). El estudio revisó 20 modelos de toma de decisiones éticos disponibles y desarrolló un modelo integrado que consta de seis pasos con preguntas y herramientas útiles que ayudan a un mejor rendimiento de cada paso.

4 Josephson Institute of Ethics. (1999)

5 Bailey, N. y Heitman, E. (2002)

The Idea Ethical

6 Decision Framework (2002)

7 Tanner, CA (2006)
El modelo se basa en seis valores llamados los "seis pilares del carácter" como la base de decisiones éticamente defendibles y el fundamento de vidas bien vividas. El modelo consta de cinco pasos cognitivos cinco pasos, con el fin de ayudar al lector a llegar a decisiones sensatas.

Se basa en los cuatro principios de la ética biomédica: respeto a la autonomía, no maleficencia, beneficencia y conducta. También toma en cuenta el código de ética de la profesión.

El marco IDEA (Identificar, determinar, explorar opciones, actuar) consiste en una serie de cuatro pasos e incorpora cinco condiciones identificadas como importantes. El propósito de IDEA como marco de decisión ética es proveer un proceso paso a paso para guiar a los proveedores del cuidado de la salud y a los administradores para trabajar con los asuntos éticos que se encuentran en la prestación de los servicios de salud. Este marco aborda dos tipos de decisiones éticas: la clínica y la organizacional. Las decisiones de ética clínica son aquellas que impactan a pacientes o miembros del staff y se enfocan en valores individuales. Las organizacionales implican grupos de pacientes o miembros.

Propone un modelo de juicio clínico como síntesis de un gran cuerpo de literatura en el tema. El proceso incluye 4 pasos y destaca el papel de los antecedentes, el contexto de la situación y la relación de las enfermeras con sus pacientes como aspecto fundamental, así como la percepción del problema, la interpretación de los resultados y la reflexión sobre la respuesta. 
Basado en el modelo de los cuatro principios, es un método evaluación ética fundamentado, procedimiento ágil de toma de decisiones. Traza un ideal ético que sirve de patrón moral para las

8 Gracia, D. (2009) acciones tanto de su planteamiento como consecuencias (ética mixta), introduce una jerarquía de los valores y propone deliberación como instrumento para aproximación a la verdad a base de integrar perspectivas distintas y complementarias: I Hechos II Valores III Deberes.

9 PLUS: The decision Making Process

Modelo de seis pasos como guía para orientar la toma de decisiones.

MER (Medical Ethical Reasoning): identifica los componentes esenciales en toma de decisiones éticas. El modelo representa adicionalmente una interacción entre los componentes:

1. Conocimiento: conocimiento médico pertinente a la intervención y conocimiento ético relevante Tsai, TC \& Harasym, implicado en la práctica médica específica.

PH. (2010)

2. estrategia de razonamiento cognitivo: incluyendo la identificación del problema, la reunión de información, la toma de decisiones, la planeación y la acción.

3. Actitudes: justificación del uso apropiado de principios, leyes o valores para racionalizar la decisión y modificar acciones.

11 Park, E.J. (2011)

Es un modelo integrado que consta de seis pasos con preguntas y herramientas útiles que ayudan a un mejor rendimiento de cada paso.

Fuente: elaboración propia con base en la revisión de la literatura.

Tabla 2. Pasos cognitivos de los modelos de toma de decisiones éticas

\begin{tabular}{|c|c|c|c|c|c|c|c|c|c|c|c|}
\hline N..$^{\circ}$ & Autor (es) & Síntesis de los pasos cognitivos & 1 & 2 & 3 & 4 & 5 & 6 & 7 & 8 & 9 \\
\hline 1 & $\begin{array}{l}\text { Thompson, J. } \\
\text { (1985) }\end{array}$ & $\begin{array}{l}\text { 1. Determinar el problema clínico y el problema ético; } \\
\text { 2. Buscar información adicional para clarificar la situación; } \\
\text { 3. Definir los actores involucrados en la decisión; } \\
\text { 4. Identificar y jerarquice las acciones junto con los } \\
\text { resultados anticipados; } 5 \text {. Definir un curso de acción; } \\
\text { 6. Evaluar los resultados de la decisión. }\end{array}$ & $x$ & $x$ & $X$ & $x$ & & $x$ & & & $x$ \\
\hline 2 & $\begin{array}{l}\text { Grundstein- } \\
\text { Amado, R. (1991) }\end{array}$ & $\begin{array}{l}\text { 1. Identificación del problema; 2. Reunión de la } \\
\text { información; } 3 \text {. Identificación de los asuntos éticos; } \\
\text { 4. Identificación de las alternativas existentes; } \\
\text { 5. Identificación de las consecuencias; } 6 \text {. Determinación } \\
\text { de la opción; 7. Justificación de la acción. }\end{array}$ & $x$ & $x$ & $x$ & $x$ & $x$ & $x$ & $x$ & & \\
\hline 3 & $\begin{array}{l}\text { Greipp, M. E. } \\
\text { (1992) }\end{array}$ & $\begin{array}{l}\text { 1. Identificación de un problema ético; } 2 \text {. Recolección de la } \\
\text { información; } 3 \text {. Desarrollo de alternativas para el análisis } \\
\text { y la comparación; } 3 \text {. Selección de las mejores alternativas } \\
\text { y justificación; } 4 \text {. Desarrollo de estrategias para poner en } \\
\text { práctica la decisión; } 5 \text {. Evaluar los efectos de la decisión. }\end{array}$ & $x$ & $x$ & & $x$ & & $x$ & & $x$ & $x$ \\
\hline 4 & $\begin{array}{l}\text { Josephson } \\
\text { Institute of Ethics } \\
\text { (1999) }\end{array}$ & $\begin{array}{l}\text { Clarificar, evaluar, decidir, implementar, monitorear y } \\
\text { modificar. }\end{array}$ & $x$ & $x$ & & & & $x$ & & $x$ & $x$ \\
\hline 5 & $\begin{array}{l}\text { Bailey, N. y } \\
\text { Heitman, E. (2002) }\end{array}$ & $\begin{array}{l}\text { 1. Reconocer e identificar el problema ético; } 2 \text {. Identificar } \\
\text { información importante; } 3 \text {. Identificar códigos } \\
\text { profesionales relevantes y principios en conflicto; } \\
\text { 4. Identificar posibles cursos de acción y sus resultados; } \\
\text { 5. Elegir el mejor curso de acción y actúe en coherencia; } \\
\text { 6. Evalúe sus acciones. }\end{array}$ & $x$ & $x$ & $x$ & $x$ & & $x$ & & & $\mathrm{x}$ \\
\hline
\end{tabular}




\begin{tabular}{|c|c|c|c|c|c|c|c|c|c|c|c|}
\hline N. ${ }^{\circ}$ & Autor (es) & Síntesis de los pasos cognitivos & 1 & 2 & 3 & 4 & 5 & 6 & 7 & 8 & 9 \\
\hline 6 & $\begin{array}{l}\text { Idea Ethical } \\
\text { Decision } \\
\text { Framework (2002) }\end{array}$ & $\begin{array}{l}\text { 1. Identifique los hechos; } 2 \text {. Determine los principios éticos } \\
\text { relevantes; 3. Explore las opciones; } 4 \text {. Actue. }\end{array}$ & $X$ & & $x$ & $X$ & & & & $x$ & \\
\hline 7 & Tanner, CA. (2006) & 1. Notar; 2. Interpretar; 3. Respuesta; 4. Reflexión. & $X$ & $X$ & & $X$ & & & & & $X$ \\
\hline 8 & Gracia, D. (2009) & $\begin{array}{l}\text { 1. Aclaración de dudas; } 2 \text {. Identificación de problemas } \\
\text { y valores en conflictos; } 3 \text {. Deliberación y propuestas de } \\
\text { cursos de acción; } 4 \text {. Elección del curso óptimo; } 5 \text {. Prueba } \\
\text { de legalidad y publicidad }\end{array}$ & & $X$ & $x$ & $X$ & & $X$ & $X$ & & \\
\hline 9 & $\begin{array}{l}\text { PLUS } 1 \text { : The decision } \\
\text { Making Process }\end{array}$ & $\begin{array}{l}\text { 1. Defina el problema; } 2 \text {. Identifique las alternativas } \\
\text { disponibles; } 3 \text {. Evalúe las alternativas identificadas; } \\
\text { 4. Tome la decisión; } 5 \text {. Implemente la decisión. }\end{array}$ & $x$ & & & $X$ & & $x$ & & $x$ & \\
\hline 10 & $\begin{array}{l}\text { Tsai, TC; Harasym, } \\
\text { PH (2010) }\end{array}$ & $\begin{array}{l}\text { 1. Identificación del problema y reunión de información; } \\
\text { 2. Toma de la decisión; 3. Planteamiento; } 4 \text {. Acción. }\end{array}$ & $X$ & & & $X$ & $X$ & & $x$ & $x$ & \\
\hline 11 & Park, E. J. (2011) & $\begin{array}{l}\text { 1. Percepción del problema ético; } 2 \text {. Recolección de la } \\
\text { información; } 3 \text {. Desarrollar alternativas, analizarlas y } \\
\text { compararlas; } 4 \text {. Seleccione las mejores alternativas y } \\
\text { justifique su decisión; } 5 \text {. Desarrollar estrategias para } \\
\text { implementar la acción elegida; } 6 \text {. Evaluar los efectos } \\
\text { de la decisión. }\end{array}$ & $X$ & $X$ & & $X$ & & $X$ & & $X$ & $x$ \\
\hline
\end{tabular}

Fuente: elaboración propia con base en la revisión de la literatura. Se marcaron con una equis los pasos identificados en cada modelo. A continuación, se identifica cada número con el paso cognitivo correspondiente: 1) identificación del problema; 2 ) recolección de información; 3) marco ético; 4) planteamiento y exploración de cursos de acción; 5) exploración de consecuencias; 6) elegir un curso de acción; 7) justificación del curso de acción; 8) actuar conforme a la decisión; y 9) monitoreo y seguimiento de la acción.

\section{Clasificación de los modelos de toma de decisiones éticas}

Según Schwartz, los modelos de toma decisiones éticas pueden clasificarse de forma general en dos categorías: aquellos que están basados en la razón y aquellos que no están basados en la razón (Schwartz, 2016). Los primeros asumen que el proceso de razonamiento moral domina el núcleo del modelo, dando lugar al juicio moral. Los segundos suponen que tanto la intuición como la emoción dominan el proceso de juicio moral, en el que la razón juega un papel secundario.

Según esta clasificación, los modelos analizados en esta revisión estuvieron centrados en una toma de decisiones éticas basada en la razón.

\section{Toma de decisiones éticas}

Se refiere al proceso completo de toma de decisiones que inicia con el reconocimiento del asunto ético e implica el razonamiento moral y las decisiones (Numminen y Leino-Kilpi, 2007).
Un dilema o problema ético es una situación que plantea un conflicto moral, en el que el agente se encuentra ante una encrucijada moral, es decir: tiene que elegir entre dos alternativas, pero ninguna de ellas está libre de problemas éticos (Ferrer y Álvarez, 2005).

Estudios sobre la toma de decisiones éticas en ciencias de la salud (Garret, 2005 y Grundstein Amado, 2001) han encontrado que, en ese contexto, las decisiones éticas se tomaron de forma simple, omitiendo aspectos relevantes del proceso y sin seguir un patrón definido.

Los hallazgos de una investigación con estudiantes de optometría (Molina, 2017) sugieren que su toma de decisiones bioéticas en la práctica clínica y frente al planteamiento de un caso real se hizo con base en la intuición moral.

Hallazgos similares se obtuvieron en un estudio con enfermeras (Alba, 2016) cuya respuesta nuclear para la toma de decisiones éticas fue la emocional $y$, en segundo lugar, la intuitiva - experiencial o la analítica- racional.

1 Acrónimo para faclitar la nemotecnia: $\mathrm{P}$, políticas; L, legal; U, universal; S, self, yo. 
La revisión de investigaciones y teorías sobre toma de decisiones éticas (Zollo, Pellegrini y Ciappei, 2017) han sugerido que la intuición moral es un antecedente de este proceso que debe ser articulado con el razonamiento moral tradicional o el proceso deliberativo o intencional.

En efecto, Epstein y Narváez proponen que, en el funcionamiento moral, la razón y la intuición se relacionan de manera muy importante, siendo el sistema intuitivo el que actúa más rápidamente ante el estímulo y el racional el que discierne las opciones y ajusta la tendencia de respuesta, corrigiéndola en caso de que no sea apropiada (Epstein, 2008, y Narváez, 2010).

Así mismo, Zhong considera que la valoración moral es un proceso racional y a la vez metafórico, informado por las emociones y el afecto y facilitado por cambios corporales que se denominan marcadores somáticos (Zhong, 2011).

Narváez propone que más que una reacción visceral, la intuición se apoya en grandes bancos de información de conocimiento implícito que las personas han adquirido de manera inconsciente (Narváez, 2010). Como se citó en Narváez (2010), este es un saber "tácito" que permite la interpretación rápida de los fenómenos y facilita la predicción con base en la comprensión de la causalidad.

Por consiguiente, el razonamiento moral se desarrolla a partir del conocimiento tácito al explícito pasando por una serie de procesos en los que las intuiciones inicialmente inexpresables se convierten en razones articuladas, de tal manera que los sistemas racional e intuitivo cooperan en la toma de decisiones. De esta manera, la deliberación es una especie de puente entre estos dos sistemas (Narváez, 2010), y posibilita su integración para una decisión justificada.

Las ideas expuestas en los apartes anteriores y las nuevas tendencias en el planteamiento de modelos de toma de decisiones éticas (Schwartz, 2016) sugieren que existe un "proceso dual" que involucra dos etapas o un proceso de "dos sistemas" mediante el cual existe una interacción concurrente entre la intuición (impulsiva) y la razón (reflexiva) que conduce al juicio moral. Estos resultados se utilizaron para hacer el planteamiento de un modelo de toma de decisiones bioéticas (TDB), proceso completo en el que el agente reconoce una cuestión bioética, hace un análisis y ponderación de opciones, toma la decisión y la justifica.

\section{Modelo de Integración Razón Emoción para la Toma de Decisiones Bioéticas en Ciencias de la Salud (MIRE)}

El esquema propuesto para la toma de decisiones bioéticas tiene las siguientes características:

1. Busca la integración razón-emoción apelando a la deliberación como elemento integrador. Callahan afirma que, dentro de la "lucha" que implica la decisión ética, la meta es conseguir un equilibrio reflexivo emotivamente fundado, en el que los sistemas están integrados y se analizan las alternativas para tomar una decisión "con todo el corazón” (Callahan, 1988). En este proceso, constantemente se deben tomar en cuenta los criterios racionales y las emociones, con el fin de evaluar la pertinencia de las respuestas.

2. Da a cada individuo la posibilidad de aplicar diferentes marcos éticos en la toma de decisiones. Según Gibson, las teorías éticas proveen una aproximación estructurada al razonamiento moral, dando lugar a una toma de decisiones completa y razonada que va más allá de la propia opinión. La teoría ética y el razonamiento no resuelven los problemas éticos, pero proveen un marco para estructurarlos y clarificarlos (Gibson, 1993).

Christensen, nos dice que introducirse en la diversidad de perspectivas éticas estimula el pensamiento crítico sobre la adecuada aplicación de estas teorías a problemas reales para idear un curso de acción moral (Christensen, 1997).

3. Sintetiza e integra los principales pasos cognitivos encontrados en los once modelos revisados en cinco fases esenciales: comprender el problema, ponderar y balancear alternativas de solución, elegir y justificar la decisión, implementar la decisión y evaluar de la decisión.

4. Busca promover particularmente el proceso enseñanza aprendizaje de toma de decisiones en el aula, aunque su uso no se limita a ese contexto y puede aplicarse en el entorno clínico. 
5. Incorpora elementos relacionados con el desarrollo de la competencia ética, que, según Balkevicius, supone tres aspectos fundamentales que comprenden diferentes habilidades: cognitivo, afectivo o imaginación moral y metacognitivo. El primero implica habilidades como la identificación de los problemas morales y la toma de decisiones mediante la selección de una opción y su justificación. El segundo aspecto está relacionado con habilidades como la reflexión sobre las emociones, sentimientos e intereses del otro y la capacidad para pronosticar los escenarios posibles de acuerdo con la situación actual. De otra parte, el tercer aspecto incluye la reflexión y justificación del trabajo en equipo en la organización, la destreza para describir el proceso de búsqueda de la información y la capacidad para justificar los asuntos sobre el trabajo (Balkevicius, 2012).

6. Aporta elementos para trabajar el pensamiento crítico, promoviendo la diferenciación del pensamiento racional y la reacción emocional. Como se citó en Millman, los pensadores críticos deben ser conscientes de sus propios sentimientos, emociones y motivaciones y las de los demás (Millman, 1988).
Cabe anotar que el modelo propuesto busca orientar la toma de decisiones y no dar respuestas correctas a preguntas éticas.

El primer paso para la toma de decisiones es la identificación o percepción del problema, es decir, la conciencia sobre la existencia de una cuestión bioética. Según Weaver, este reconocimiento e interpretación de la dimensión ética de una situación de cuidado se denomina sensibilidad ética y es un aspecto crucial para iniciar el proceso de TDB (Weaver, 2007).

Dado que el MIRE busca la integración razón -emoción, el objetivo de esta fase es atender a las intuiciones iniciales manteniendo la calma y la mente abierta para identificar cuál es la situación que requiere resolverse, reunir toda la información relevante que esté disponible, buscar la información faltante con el fin de ampliar el entendimiento del problema y tomar en cuenta los intereses de todos los que serán afectados por la decisión (ver tabla 3).

El segundo paso para la TDB consiste en identificar, ponderar y balancear las alternativas de solución. Se debe examinar el dilema moral/ético mediante el análisis, contemplación, elección y evaluación de las razones para una acción dada,

Figura 1. MIRE: Modelo de Integración Razón Emoción para la Toma de Decisiones Bioéticas en Ciencias de la Salud

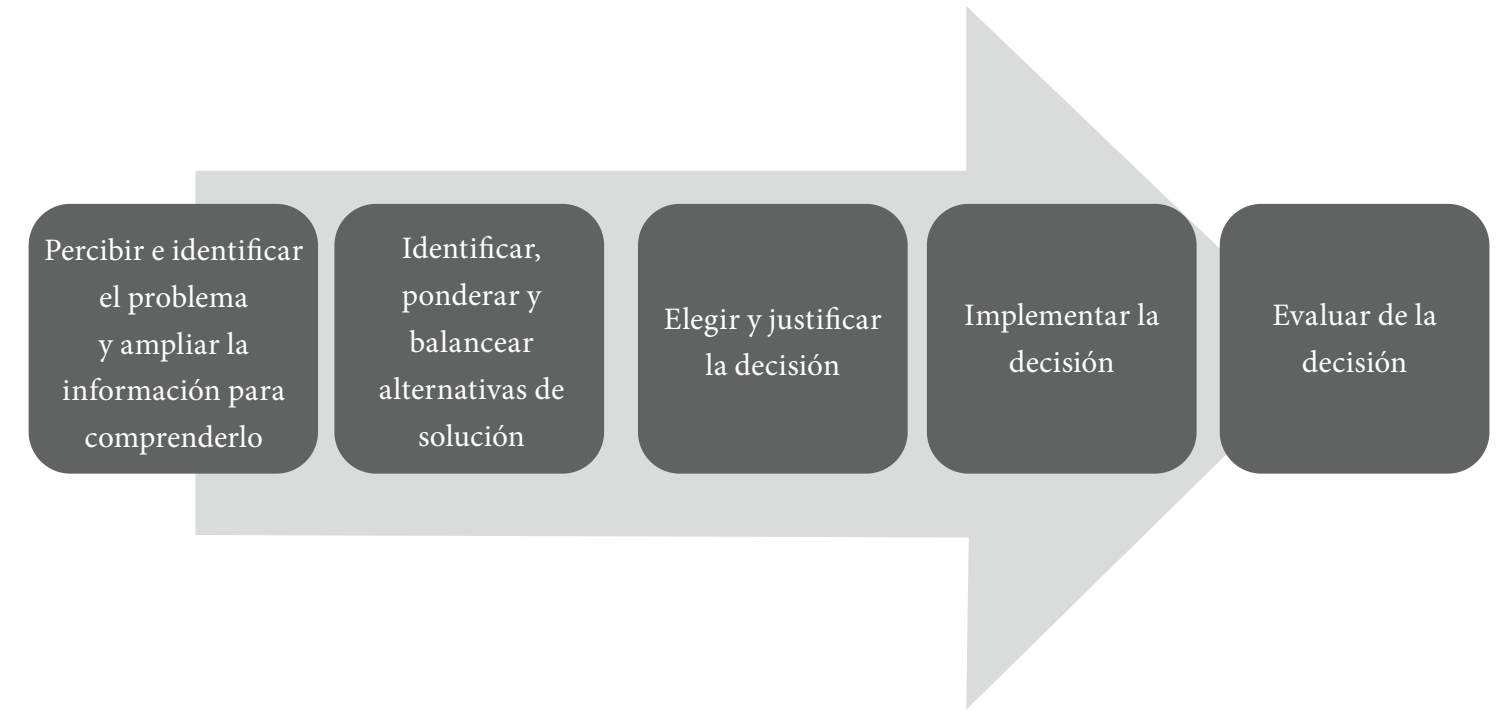

Fuente: elaboración propia. 
Tabla 3. Paso 1. Comprender el problema

\begin{tabular}{|c|c|c|c|c|}
\hline Objetivos & Tome mejores decisiones & $\begin{array}{l}\text { Preguntas } \\
\text { orientadoras }\end{array}$ & Aspectos para tomar en cuenta & Tareas \\
\hline \multirow{2}{*}{$\begin{array}{l}\text { Percibir e } \\
\text { identificar el } \\
\text { problema }\end{array}$} & \multirow{2}{*}{$\begin{array}{l}\text { Conserve la calma y tómese un } \\
\text { tiempo para reflexionar sobre el } \\
\text { caso, para comenzar a entender } \\
\text { el problema y todos los aspectos } \\
\text { que lo rodean. } \\
\text { Identifique sus sentimientos } \\
\text { y emociones y concédales } \\
\text { importancia pero mantenga la } \\
\text { mente abierta. }\end{array}$} & $\begin{array}{l}\text { ¿Qué es lo que se } \\
\text { debe resolver desde el } \\
\text { punto de vista clínico? }\end{array}$ & $\begin{array}{l}\text { La anamnesis, signos, síntomas, } \\
\text { pruebas clínicas y exámenes } \\
\text { complementarios, diagnóstico } \\
\text { y pronóstico sirven como } \\
\text { orientación para identificar el } \\
\text { problema clínico. }\end{array}$ & $\begin{array}{l}\text { Describa el } \\
\text { problema clínico }\end{array}$ \\
\hline & & $\begin{array}{l}\text { ¿Qué es lo que se } \\
\text { debe resolver desde el } \\
\text { punto de vista ético? }\end{array}$ & $\begin{array}{l}\text { Verifique si está en juego el } \\
\text { bienestar, los derechos o deberes } \\
\text { de alguien y qué principios están } \\
\text { oposición o tensión. }\end{array}$ & $\begin{array}{l}\text { Describa el } \\
\text { problema ético }\end{array}$ \\
\hline \multirow{4}{*}{$\begin{array}{l}\text { Analizar y buscar } \\
\text { información } \\
\text { para ampliar el } \\
\text { entendimiento } \\
\text { del problema }\end{array}$} & $\begin{array}{l}\text { Deténgase y trate de observar } \\
\text { la situación como un todo, } \\
\text { considere todos los aspectos que } \\
\text { intervienen o pueden intervenir. }\end{array}$ & $\begin{array}{l}\text { ¿Qué información está } \\
\text { disponible? }\end{array}$ & $\begin{array}{l}\text { Analice detalladamente el caso y } \\
\text { tome nota de todos los aspectos } \\
\text { que surjan. }\end{array}$ & $\begin{array}{l}\text { Haga el } \\
\text { listado de la } \\
\text { información } \\
\text { disponible. }\end{array}$ \\
\hline & $\begin{array}{l}\text { Sea sensible a las necesidades } \\
\text { del paciente y reflexione } \\
\text { detenidamente sobre las } \\
\text { personas o grupos que serán } \\
\text { afectados por su decisión. }\end{array}$ & $\begin{array}{l}\text { ¿Qué información } \\
\text { necesito para resolver } \\
\text { el problema? }\end{array}$ & $\begin{array}{l}\text { Identifique los datos y hechos } \\
\text { que le ayudan o ayudarían a } \\
\text { comprender el problema. }\end{array}$ & $\begin{array}{l}\text { Haga un } \\
\text { listado de la } \\
\text { información } \\
\text { que requiere. }\end{array}$ \\
\hline & $\begin{array}{l}\text { Mantenga en mente el problema } \\
\text { original y no se } \\
\text { desvíe o desenfoque. }\end{array}$ & $\begin{array}{l}\text { ¿Tengo toda la } \\
\text { información que } \\
\text { requiero para } \\
\text { solucionar el } \\
\text { problema? }\end{array}$ & $\begin{array}{l}\text { Contraste los listados de la } \\
\text { información disponible y la } \\
\text { información que requiere. }\end{array}$ & $\begin{array}{l}\text { Verifique si la } \\
\text { información es } \\
\text { suficiente. }\end{array}$ \\
\hline & $\begin{array}{l}\text { Responde la afirmación: este } \\
\text { problema sería más fácil de } \\
\text { resolver si supiera... }\end{array}$ & $\begin{array}{l}\text { ¿Qué otra información } \\
\text { requiero y en donde } \\
\text { puedo buscarla? }\end{array}$ & $\begin{array}{l}\text { Recuerde que entre mejor } \\
\text { informado esté mejor será su } \\
\text { comprensión del problema. } \\
\text { Infórmese bien y busque fuentes } \\
\text { que tengan credibilidad. }\end{array}$ & $\begin{array}{l}\text { Haga un listado } \\
\text { teniendo en } \\
\text { cuenta la } \\
\text { información } \\
\text { requerida y la } \\
\text { fuente. }\end{array}$ \\
\hline
\end{tabular}

Fuente: elaboración propia.

con el fin de determinar cuál de las alternativas es la mejor desde el punto de vista moral (Lanik y Webb, 1989). Mitchel, R. C. afirma que las decisiones éticas son complejas debido a que implican múltiples alternativas, consecuencias a largo plazo y resultados inciertos que combinan beneficios, costos económicos, legales, sociales e implicaciones personales diversas. Así como objetivos e intereses variados y a menudo opuestos (Mitchel, R. C., 2009).

El objetivo de esta fase de la toma de decisiones es identificar las alternativas de solución, ponderarlas y balancearlas evaluando los resultados y/o consecuencias de cada una, procurando mantenerse imparcial, evitando sesgos y tomando en cuenta todos los puntos de vista (ver tabla 4).
El tercer paso es elegir y justificar la decisión. La justificación tiene lugar cuando el tomador de la decisión defiende el curso de la acción elegido, evaluándolo moralmente y reflexionando sobre el: ¿cómo hice esta decisión? ¿Qué normas y valores estuvieron implicados? ¿En qué extensión estuvo determinada mi decisión por la tradición, factores sociales, culturales o socioeconómicos y demás? (De Lange, 2012).

El objetivo de esta fase de la toma de decisiones es definir el curso óptimo de acción, teniendo en cuenta las evidencias y siendo ecuánime, justo y veraz. Reúna los elementos necesarios para construir un argumento que justifique su decisión (ver tabla 5). 
Tabla 4. Paso 2. Identificar, ponderar y balancear alternativas de solución

\begin{tabular}{|c|c|c|c|c|}
\hline Objetivos & Tome mejores decisiones & $\begin{array}{l}\text { Preguntas } \\
\text { orientadoras }\end{array}$ & $\begin{array}{l}\text { Aspectos a tomar en } \\
\text { cuenta }\end{array}$ & Tareas \\
\hline \multirow[t]{2}{*}{$\begin{array}{l}\text { Reconocer, analizar y sopesar } \\
\text { cursos de acción }\end{array}$} & \multirow{2}{*}{$\begin{array}{l}\text { Sea sensible y manténgase } \\
\text { alerta frente a los posibles, } \\
\text { sesgos, prejuicios o } \\
\text { limitaciones de su punto de } \\
\text { vista. } \\
\text { Revise sus creencias a la luz de } \\
\text { las evidencias. }\end{array}$} & $\begin{array}{l}\text { ¿Qué alternativas } \\
\text { de solución podría } \\
\text { plantear? }\end{array}$ & $\begin{array}{l}\text { Tome en cuenta que } \\
\text { existen diversas teorías } \\
\text { éticas para guiarlo, que } \\
\text { pueden llevar a plantear } \\
\text { alternativas diversas. }\end{array}$ & $\begin{array}{l}\text { Haga un } \\
\text { listado de las } \\
\text { alternativas }\end{array}$ \\
\hline & & $\begin{array}{l}\text { ¿Existe alguna } \\
\text { otra alternativa de } \\
\text { solución? }\end{array}$ & $\begin{array}{l}\text { Verifique si dejó de } \\
\text { contemplar alguna } \\
\text { alternativa de solución. }\end{array}$ & $\begin{array}{l}\text { Refine su lista } \\
\text { de alternativas }\end{array}$ \\
\hline $\begin{array}{l}\text { Evaluar los resultados y/0 } \\
\text { consecuencias de cada uno } \\
\text { de los cursos de acción }\end{array}$ & $\begin{array}{l}\text { Póngase en el lugar de todos } \\
\text { los afectados e imagine lo que } \\
\text { sienten y quieren. } \\
\text { Sea consciente de sus propios } \\
\text { sentimientos, emociones } \\
\text { y motivaciones y las de los } \\
\text { demás. } \\
\text { No pierda de vista su } \\
\text { perspectiva pero tenga en } \\
\text { cuenta la de los otros. }\end{array}$ & $\begin{array}{l}\text { ¿Cuáles son los } \\
\text { resultados y/o } \\
\text { consecuencias de cada } \\
\text { decisión? }\end{array}$ & $\begin{array}{l}\text { Determine las } \\
\text { consecuencias positivas } \\
\text { y negativas de cada } \\
\text { alternativa en el corto } \\
\text { mediano y largo plazo. }\end{array}$ & $\begin{array}{l}\text { Haga un } \\
\text { listado de las } \\
\text { consecuencias } \\
\text { en frente } \\
\text { de cada } \\
\text { alternativa de } \\
\text { solución. }\end{array}$ \\
\hline
\end{tabular}

Fuente: elaboración propia.

Tabla 5. Paso 3. Elegir y justificar la decisión

\begin{tabular}{|c|c|c|c|c|}
\hline Objetivos & Tome mejores decisiones & $\begin{array}{l}\text { Preguntas } \\
\text { orientadoras }\end{array}$ & $\begin{array}{c}\text { Aspectos para tomar en } \\
\text { cuenta }\end{array}$ & Tareas \\
\hline $\begin{array}{l}\text { Priorizar los } \\
\text { cursos de acción } \\
\text { eligiendo el que } \\
\text { se considera } \\
\text { óptimo. }\end{array}$ & $\begin{array}{l}\text { Tome en cuenta todos los cursos } \\
\text { de acción surgidos a la luz de las } \\
\text { evidencias, incluso aquellos que } \\
\text { van en contra de lo que usted } \\
\text { piensa o aquellos a los que no se } \\
\text { ha prestado suficiente atención. } \\
\text { Es momento de contrastar su } \\
\text { intuición inicial con los hallazgos } \\
\text { y evidencias. Sea honesto, justo } \\
\text { y veraz. }\end{array}$ & $\begin{array}{l}\text { ¿Cuál es el mejor curso } \\
\text { de acción? }\end{array}$ & $\begin{array}{l}\text { No olvide que debe elegir } \\
\text { entre las alternativas que sean } \\
\text { éticamente justificables. }\end{array}$ & $\begin{array}{l}\text { Escriba el } \\
\text { curso de acción } \\
\text { elegido. }\end{array}$ \\
\hline \multirow[t]{2}{*}{$\begin{array}{l}\text { Plantear buenas } \\
\text { razones que } \\
\text { apoyan esta } \\
\text { elección. }\end{array}$} & \multirow{2}{*}{$\begin{array}{l}\text { Argumente. No pierda de vista } \\
\text { el curso de acción elegido, esa } \\
\text { es la conclusión. Ahora, plantee } \\
\text { afirmaciones que apoyen o } \\
\text { soporten esta conclusión. Indique } \\
\text { por qué considera que es correcto } \\
\text { tomar este curso de acción desde } \\
\text { el punto de vista moral. Trate } \\
\text { de elaborar su argumento de la } \\
\text { forma más detallada posible. Evite } \\
\text { la ambigüedad. }\end{array}$} & $\begin{array}{l}\text { ¿Por qué esta decisión } \\
\text { es mejor que otras? }\end{array}$ & $\begin{array}{l}\text { Verifique todas las razones. } \\
\text { No pierda de vista las } \\
\text { evidencias, teorías, reglas } \\
\text { / principios, códigos } \\
\text { profesionales, creencias, } \\
\text { valores, pautas, consecuencias, } \\
\text { casos comparables y } \\
\text { experiencias previas (ver } \\
\text { teorías éticas, Anexo I). }\end{array}$ & Haga una lista \\
\hline & & $\begin{array}{l}\text { ¿Por qué esta decisión } \\
\text { es la mejor decisión } \\
\text { que puedo tomar? }\end{array}$ & $\begin{array}{l}\text { No olvide enunciar su decisión } \\
\text { y las razones por las que } \\
\text { considera que es correcta } \\
\text { desde el punto de vista ético. } \\
\text { Sea concreto y evite la } \\
\text { ambigüedad. }\end{array}$ & $\begin{array}{l}\text { Elabore un } \\
\text { argumento } \\
\text { moral que } \\
\text { justifique su } \\
\text { decisión. }\end{array}$ \\
\hline
\end{tabular}

Fuente: elaboración propia. 
El cuarto paso es implementar la decisión. Esto implica la planeación y anticipación de cualquier barrera que puede interferir con la implementación del curso de acción (Francis, 2015). El objetivo de esta fase de la toma de decisiones, es hacer un plan de acción definiendo estrategias para su implementación, teniendo en cuenta los posibles obstáculos y definiendo metas y plazos concretos en el tiempo (ver tabla 6).

El quinto paso es evaluar la decisión. El objetivo es identificar qué tan eficaz fue el curso de acción elegido como decisión para resolver la situación y qué oportunidades de mejoramiento surgieron a partir de la experiencia vivida para cualificar la TDB (ver tabla 5).

\section{Utilización del modelo}

Nora, Deodato, Vieira y Zoboli concluyen que para llegar a una decisión prudente se necesita experiencia. Por lo tanto, es esencial que los profesionales desarrollen habilidades y competencia ética para ayudar a manejar la incertidumbre y reducir la angustia que causa el proceso ético de toma de decisiones (Nora, Deodato, Vieira y Zoboli, 2016).

Tabla 6. Paso 4. Implementar la decisión

\begin{tabular}{|c|c|c|c|c|}
\hline Objetivos & $\begin{array}{l}\text { Tome mejores } \\
\text { decisiones }\end{array}$ & $\begin{array}{l}\text { Preguntas } \\
\text { orientadoras }\end{array}$ & Aspectos para tomar en cuenta & Tareas \\
\hline \multirow{2}{*}{$\begin{array}{l}\text { Establecer las } \\
\text { estrategias que se } \\
\text { van a desplegar } \\
\text { para implementar } \\
\text { la decisión }\end{array}$} & \multirow{2}{*}{$\begin{array}{l}\text { Reflexione detenidamente } \\
\text { sobre los aspectos que } \\
\text { influencian la puesta } \\
\text { en marcha de la acción. } \\
\text { Identifique los pros y } \\
\text { contras. Idee formas de } \\
\text { superar los obstáculos } \\
\text { que se podrían presentar. } \\
\text { Establezca metas y plazos. }\end{array}$} & $\begin{array}{l}\text { ¿Cómo puedo poner en } \\
\text { práctica mi decisión? }\end{array}$ & $\begin{array}{l}\text { Tenga en cuenta todos los aspectos: } \\
\text { personales, interpersonales, } \\
\text { contextuales y éticos. De cara a } \\
\text { la implementación de la decisión } \\
\text { responda las siguientes preguntas: } \\
\text { ¿qué?, ¿quién?, ¿cómo?, ¿dónde?, } \\
\text { ¿cuándo? Y ¿por qué? }\end{array}$ & $\begin{array}{l}\text { Realizar un plan de } \\
\text { acción con metas } \\
\text { concretas }\end{array}$ \\
\hline & & $\begin{array}{l}\text { ¿Existen obstáculos para } \\
\text { poner en práctica mi } \\
\text { decisión? ¿Cómo puedo } \\
\text { superarlos? }\end{array}$ & $\begin{array}{l}\text { Tenga en cuenta todos los aspectos: } \\
\text { personales, interpersonales, } \\
\text { contextuales y éticos. }\end{array}$ & $\begin{array}{l}\text { Haga un listado de } \\
\text { los obstáculos y } \\
\text { como superar cada } \\
\text { uno de ellos. }\end{array}$ \\
\hline
\end{tabular}

Fuente: elaboración propia.

Tabla 7. Paso 5. Evaluar de la decisión

\begin{tabular}{|c|c|c|c|c|}
\hline Objetivos & Tome mejores decisiones & $\begin{array}{l}\text { Preguntas } \\
\text { orientadoras }\end{array}$ & $\begin{array}{l}\text { Aspectos para } \\
\text { tomar en cuenta }\end{array}$ & Tareas \\
\hline \multirow{2}{*}{$\begin{array}{l}\text { Identificar el alcance y } \\
\text { efectividad de la decisión } \\
\text { y las oportunidades } \\
\text { de mejoramiento } \\
\text { del proceso seguido, } \\
\text { aprendizaje que } \\
\text { redundará en una } \\
\text { cualificación del mismo. }\end{array}$} & \multirow{2}{*}{$\begin{array}{l}\text { Haga un seguimiento a } \\
\text { su decisión. Verifique los } \\
\text { resultados reales y compárelos } \\
\text { con los resultados probables } \\
\text { que usted definió. El balance } \\
\text { de estos aspectos señalará } \\
\text { algunas cuestiones que usted } \\
\text { omitió y eran importantes. La } \\
\text { experiencia vivida es importante } \\
\text { como punto de referencia para } \\
\text { tomar decisiones en adelante. }\end{array}$} & $\begin{array}{l}\text { ¿Cuáles son los resultados } \\
\text { de la decisión-acción a corto, } \\
\text { mediano y largo plazo? }\end{array}$ & $\begin{array}{l}\text { Tome en cuenta los } \\
\text { pros y contras }\end{array}$ & $\begin{array}{l}\text { Haga una lista } \\
\text { de resultados }\end{array}$ \\
\hline & & $\begin{array}{l}\text { ¿Qué aspectos puedo tener } \\
\text { en cuenta para hacerlo } \\
\text { mejor en una próxima } \\
\text { oportunidad? }\end{array}$ & $\begin{array}{l}\text { Tenga en cuenta } \\
\text { los aspectos que no } \\
\text { funcionaron como } \\
\text { esperaba y enuncie la } \\
\text { forma de mejorarlos }\end{array}$ & $\begin{array}{l}\text { Describa los } \\
\text { aspectos por } \\
\text { mejorar }\end{array}$ \\
\hline
\end{tabular}

Fuente: elaboración propia. 
Por tanto, dentro la formación bioética de los profesionales de la salud (DeSimone, 2016) se debe promover el desarrollo de la competencia ética, el pensamiento crítico y otras habilidades necesarias para hacer frente de forma adecuada a las etapas de la toma de decisiones bioéticas durante la formación profesional (Molina, 2017). Adicionalmente, debe fomentar la comprensión de diversas perspectivas éticas para que pueda aplicarlas de forma crítica a problemas reales dentro del proceso de toma de decisiones bioéticas (Christensen, 1997). La tabla 8 enumera algunas teorías éticas, su definición, máxima y algunos aspectos clave para su aplicación en la toma de decisiones bioéticas, con lo que se constituye en una herramienta complementaria al uso del MIRE.

Tabla 8. Resumen de algunas teorías éticas y su aplicación en la tdb

\begin{tabular}{|c|c|c|c|}
\hline Teoría & Definición & Máxima & $\begin{array}{c}\text { Aplicación en la toma de decisiones } \\
\text { bioéticas }\end{array}$ \\
\hline $\begin{array}{l}\text { Teleología y } \\
\text { utilitarismo. } \\
\text { Bentham y Mill }\end{array}$ & $\begin{array}{l}\text { Define el acto moral en términos de los } \\
\text { resultados o las consecuencias de ese acto } \\
\text { (Bennet-Woods, 2005). Por esta razón, son } \\
\text { clasificadas como teorías consecuencialistas. }\end{array}$ & $\begin{array}{l}\text { "Elige maximizar el bien } \\
\text { y minimizar el daño" } \\
\text { (Thomamsma, 2003). }\end{array}$ & $\begin{array}{l}\text { Las consecuencias son evaluadas en } \\
\text { términos de utilidad, costo efectividad y } \\
\text { eficiencia, de tal suerte que se cumpla el } \\
\text { mayor bien para el mayor número (Gibson, } \\
\text { 2004). Por lo tanto, ninguna acción es } \\
\text { buena o mala en sí misma. Las acciones } \\
\text { son juzgadas a la luz de sus consecuencias } \\
\text { (Bennet-Woods, 2005). }\end{array}$ \\
\hline
\end{tabular}

Se enfoca en las obligaciones vinculantes o deberes en los que la justificación de los principios y acciones son independientes de las consecuencias. En otras palabras, un acto es

Ética deontológica I. Kant correcto, si y solo si, cumple con la obligación moral primordial, e incorrecta, si viola el deber o principio moral principal (Gibson, 2004). Las personas son seres humanos que construyen su propia ley moral, deben ser valoradas por su valor intrínseco y ser tratadas como fines en sí mismos y no solamente como medios a los fines de otros (Gibson, 2004).
"Actúa de tal manera que la regla o principio rector de tu acción pueda ser una ley universal (BennetWoods, 2005).
Una acción moral es aquella que se realiza con el único fin de satisfacer una obligación moral, y la acción en sí misma solo puede ser juzgada moral a la luz de la intención detrás de ella (Bennet-Woods, 2005).
Centra su atención en el carácter como fuente de la acción moral (Bennet-Woods, 2005).

Ética de las virtudes Aristóteles Las virtudes son rasgos de carácter que predisponen a una persona a hacer lo correcto, cuando se enfrenta a una elección moral (Bennet-Woods, 2005). "se virtuoso y compórtate con excelencia para vivir una vida buena".
La conducta moral asume las buenas características de quien toma la decisión como agente moral, quien debe demostrar excelencia (areté) y comportarse de una forma adecuada, lo cual requiere del desarrollo de buenas virtudes (Mcgonigle, Mastriany Farcus, s.f.).
Se basa en cuatro principios prima facie, es decir, que deben respetarse siempre, a menos que exista una poderosa razón que justifique incumplirlos (Thomasma, 2003).

Los principios son: 1) respeto por la autonomía, Principialismo. 2) no maleficencia, 3) beneficencia y 4) justicia. Beauchamp y El conjunto de principios funciona como una Childress estructura analítica que intenta expresar normas generales de la moralidad común que son un punto de partida de la ética biomédica. Estos principios deben funcionar como guías generales para la formulación de reglas más específicas (Beauchamp y Childress, 2009).
"Los cuatro principios sirven como base para la resolución de los dilemas éticos"
Para la toma de decisiones se definen los principios en conflicto de acuerdo con el caso y se realiza la ponderación (comparación) de los mismos. Se interroga sobre cuál de ellos tiene mayor peso. Se evalúa la importancia y solidez de un principio con respecto a otro. 


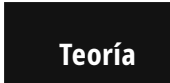

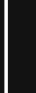

Se utiliza para designar un marco general

Pluralismo moral

(pluralismo ético) sobre cómo llegar a juicios éticos sólidos. Este enfoque utiliza un procedimiento paso a paso.
Máxima

"Para tomar una decisión ética razonada se debe considerar y aplicar la mayor cantidad de conceptos éticos, principios y teorías que sean convenientes con el fin de resolver el asunto".
Aplicación en la toma de decisiones bioéticas

Desde esta perspectiva teórica, se considera que dada la complejidad de las cuestiones éticas, problemas y dilemas, se requieren diversas teorías que permitan resolver decisiones morales difíciles (Center for the Advancement of Applied Ethics at Carnegie, 2003).
Es un intento por revisar o replantear la ética tradicional en la medida en que esta deprecia la experiencia moral de las mujeres (Tong y Williams, s.f.).

Allison Jaggar, entre otras feministas, culpa a las éticas tradicionales de dejar desatendidas a las mujeres en al menos cinco aspectos: 1 ) menor atención por los problemas e intereses de las mujeres en comparación con los hombres, 2) trivializar los asuntos morales que surgen en el ámbito privado, 3) sugerir

Ética feminista que las mujeres son menos maduras desde el punto de vista moral que los hombres, 4) sobreestimar rasgos culturalmente masculinos y subestimar rasgos culturalmente femeninos. Por último, 5) favorecer "formas masculinas de razonamiento moral que hacen hincapié en las normas, los derechos, la universalidad, e imparcialidad sobre formas "femeninas" de razonamiento moral que hacen hincapié en las relaciones, responsabilidades, particularidad, y la parcialidad. El abordaje de estas falencias han dado origen al desarrollo de una amplia variedad de enfoques (Tong y Williams, s.f.).

Es una aproximación a la ética médica del encuentro clínico. Este es un enfoque en el que se da cuenta de la identidad personal experimentada como vida-narrativa. Es una herramienta en el análisis fenomenológico de la experiencia de enfermedad con el potencial

Ética narrativa de influir en la práctica profesional (Brock, 2006).

Este enfoque busca entender los individuos en el sistema de salud mediante la captura de su esencia a través de la comprensión de las historias que definen su existencia (Weizenbluth, Sokolowski y Gordon, 2008).
"La meta es eliminar 0 al menos mejorar la opresión de cualquier grupo de personas, pero en particular las mujeres" (Tong y Williams, s. f.).
Las acciones son vistas en relación con su efecto sobre la calidad de las relaciones entre las personas, con énfasis en: las consideraciones de justicia, el concepto de cuidado, la importancia de considerar el contexto de una situación individual en la toma de decisiones médicas y en las relaciones permite que la emoción y la intuición sean factores importantes en las deliberaciones (Bennet-Woods, 2005).
"Comprende las historias y las características que conforman la identidad y el bienestar de los pacientes y los profesionales para asegurar un tratamiento ético, comunicación y toma de decisiones adecuados" (Weizenbluth, Sokolowski y Gordon, 2008).
En este enfoque, el pensamiento ético está

Ética comunitaria fundado en valores comunitarios, estándares sociales, tradiciones y consideraciones de la sociedad en general (Weizenbluth, Sokolowski y Gordon, 2008).
“Una acción es ética si está enmarcada dentro del sistema de valores compartidos por la comunidad".
La ética narrativa proporciona un marco para la toma de decisiones éticas. Este marco apoya a los profesionales de la salud para que puedan tomar una decisión ética en relación con la vida y el bienestar de una persona, tomando en cuenta las historias de ese individuo, su historia y carácter como un componente clave del proceso de toma de decisiones. Este enfoque complementa el enfoque principialista (Weizenbluth, Sokolowski y Gordon, 2008).

La clave para responder preguntas éticas y conflictos, reside en el respeto por los valores locales que demuestran la deliberación y aceptación de la comunidad local. Se debe prestar atención general y responsabilidad con los valores de la sociedad general Weizenbluth, Sokolowski y Gordon, 2008).

Fuente: elaboración propia a partir de los autores. 


\section{Conclusiones}

Los modelos de toma de decisiones éticas se plantean como referencia para guíar el proceso paso a paso. Los 11 esquemas revisados son de carácter teórico y están centrados en la toma racional de decisiones éticas, proponen un número variable de pasos cognitivos que está entre 4 a 7 y ofrecen pocas pautas para orientar el proceso de toma de decisiones éticas. Solo el esquema planteado por Grundstein-Amado ha sido probado empíricamente y ajustado con base en la investigación.

Se propone el MIRE un esquema teórico que sintetiza los pasos cognitivos para alcanzar una adecuada toma de decisiones bioéticas, mediante la integración de la razón y la emoción a través de la deliberación, la posibilidad de aplicación de diversos marcos éticos y la puesta en juego de la competencia ética y las habilidades de pensamiento crítico. Este esquema ofrece al tomador de decisiones bioéticas, pautas claras y fáciles de implementar para orientar este proceso.

Este modelo, puede constituirse en una herramienta útil para profesores y estudiantes en ciencias de la salud para la formación en materia de toma de decisiones bioéticas, en conjunto con otras estrategias que posibiliten el desarrollo de la deliberación y de las habilidades de pensamiento crítico.

\section{Referencias}

Alba, B. (2018). Factors that impact on emergency nurses' ethical decision-making ability. Nursing Ethics, 25(7), 855-866. doi: 10.1177/0969733016674769.

Arries, E. (2005). Virtue Ethics: an approach to moral dilemmas in nursing. Curationis, 28(3), 64-72. doi: https:// doi.org/10.4102/curationis.v28i3.990

Bailey, N. \& Heitman, E. (2002). An optometrist Guide to Clinical Ethics. St. Louis, Missouri: American Optometric Association. Retrieved from: https:/www.aoa. org/documents/optometrists/book.pdf

Balkevicius, M. (2012). Ethical Competence: Problem Based Learning Approach in Moral Education. Lithuanian University of Educational Sciences, Department of Education. Retrieved from: http://www.ramk.fi/loader. aspx?id=4dbd2726-fdbf-4a3b-91e9-b2798ff9ec0d

Beauchamp, T. \& Childress, J. (2009). Principles of Biomedical Ethics. New York: Oxford University Press.
Bennet-Woods, Deb. (2005). Ethics at a glance. Kantian Ethics. Regis University. Retrieved from: http://rhchp. regis.edu/HCE/EthicsAtAGlance/.

Brock, SC. (2006). Narrative Ethics. En: eLS. John Wiley \& Sons. Ltd, Chichester. Retrieved from: http://www.els.net

Callahan, S. (1988). The Role of Emotion in Ethical Decision - Making. The Hastings Center Report, 18(3), 9-14. doi: https://doi.org/10.2307/3562196

Cameron, M., Schaffer, M. \& Park, H. (2000). Nursing students' experience of ethical problems and use of ethical decision-making models. Nursing Ethics, 8(5), 432-447. doi: https://doi.org/10.1177/096973300100800507

Cassells, M., Jenkins, J., Lea, D., Calzone, K. \& Johnson, E. (2003). An ethical assessment framework for addressing global genetic issues in clinical practice. Oncology Nursing Forum, 30(3), 383-390. doi: 10.1188/03.ONF.383-390

Cerit, B. \& Dinc, L. (2012). Ethical decision making and professional behavior among nurses: A correlational Study. Nursing ethics, 20(2), 200-212. Doi: https://doi. org/10.1177/0969733012455562

Center for the Advancement of Applied Ethics at Carnegie Mellon University (2003). Retrieved from: http://www. andrew.cmu.edu/user/pm2n/PPGSite/discussion.htm

Choe, K., Song, E. \& Kang, Y. (2013). Recognizing bioethical issues and ethical qualification in nursing students and faculty in South Korea. Nursing Ethics, 20(2), 213 225. doi: https://doi.org/10.1177/0969733012472734

Christensen, J. (1997) Teaching Tutorial. Assisting the learning of ethics. Optometric Education. The Journal of the Association of Schools and Colleges of Optometry, 22(4). Retrieved from: https://journal.opted.org/files/ Volume_22_Number_4_Summer_1997.pdf

DeSimone, B. (2016). Curriculum Design to Promote the Ethical Decision-Making Competence of Accelerated Bachelor's Degree Nursing Students. SAGEOpen. January-March,1-10.doi:https://doi.org/10.1177/ 2158244016632285

Epstein, S. (2008). Intuition from the perspective of cognitive-experiential self-theory. En: H. Plessner, C. Betsch \& T. Betsch (Eds.), Intuition in judgment and decision making (pp. 23-37). New York: Laurence Earlbaum Associates/Taylor \& Francis Group.

Ferrer, J.J. y Álvarez, J.C. (2005). Para fundamentar la bioética. Teorías y paradigmas teóricos en la bioética contemporánea. 2. ${ }^{\mathrm{a}}$ ed. Bilbao: Universidad Pontificia Comillas-Ed. Desclée De Brouwer.

Francis, P.C. (2015). A Review of Contemporary Ethical Decision-Making Models for Mental Eastern Michigan University. Retrieved from: https://files.eric.ed. gov/fulltext/ED565003.pdf 
García, D.J. (2011). La deliberación moral en bioética. Interdisciplinariedad, pluralidad, especialización. Ideas y Valores, 60(147), 20-25. Recuperado de: https://revistas. unal.edu.co/index.php/idval/article/view/36771/38743.

Gracia, D. (2009). UD2 Método de la bioética. Recuperado de: http://cvb.ehu.es/open_course_ware/castellano/salud/bioetica/content/ud2_metodo_ocw_09.pdf

Garret, B. (2005). Student nurses' perceptions of clinical decision-making in the final year of adult nursing studies. Nurse Education in Practice, 5(5), 30-39. doi: https://doi. org/10.1016/j.nepr.2004.03.003

Gibson, C. (1993). Underpinnings of ethical reasoning in nursing. Journal of Advanced Nursing, 18(12). doi: https:// doi.org/10.1046/j.1365-2648.1993.18122003.

Greipp, M. (1992). Greipp's model of ethical decision making. Journal of Advanced Nursing, 17(6). doi: https://doi. org/10.1111/j.1365-2648.1992.tb01972.x

Grundstein-Amado, R. (1991). An integrative model of Clinical-ethical decision making. Theoretical $\mathrm{Me}$ dicine and bioethics, 12(2), 157-170. doi: https://doi. org/10.1007/BF00489796

Grundstein-Amado, R. (1992). Differences in ethical decision-making processes among nurses and doctors. Journal Of Advanced Nursing, 17(2), 129-137. doi: https://doi. org/10.1111/j.1365-2648.1992.tb01867

House, J., Theyyunni, N., Barnosky, A., Fuhrel-Forbis, A., Seeyave, D., Ambs, D., Fisher, J. \& Santen, S. (2015). Understanding ethical dilemmas in the emergency department: views from medical students' essays. The Journal of Emergency Medicine, 48(4), 492-498. https:// doi.org/10.1016/j.jemermed.2014.09.058

Josephson Institute of Ethics. (1999). The Seven-Step Path To Better Decisions Retrieved from: Http://Josephsoninstitute.Org/Med-4sevensteppath/

Kader, NS. (2005). Justifying Bioethical case decisions: reflective equilibrium and mid-level moral principles. Master's Thesis. University of Maryland. https://drum. lib.umd.edu/handle/1903/2404

Lanik, G. \& Webb, A. (1989). Ethical decision making for community health nurses. Journal of Community Health Nursing, 6(2), 95-102. Retrieved from: http:// www.jstor.org/stable/3427210

Mcgonigle, D., Mastrian, K. \& Farcus, N. (s.f.). Ethical Challenges in Healthcare Informatics. Retrieved from: http://www.personal.psu.edu/dxm12/n458/index.htm

Mitchel, R.C. (2009). Ethical Considerations in Decision Making. Retrieved from: http://www.csun.edu/ hfmgt001/ ethicalFr.htm

Molina Montoya, N. (2016). Formación bioética en ciencias de la salud. Ciencia y Tecnología para la Salud Visual y Ocular, 14(1), 117-132. https://doi.org/10.19052/sv.3544
Molina Montoya, N. (2016). Problemas bioéticos percibidos por los estudiantes que desarrollan su práctica en la Clínica de Optometría de la Universidad de La Salle. Ciencia y Tecnología para la Salud Visual y Ocular, 14(2), 53-60. https://doi.org/10.19052/sv.3837

Molina, M, N. (2017). Toma de decisiones bioéticas en optometría clínica. Bogotá: Ediciones Unisalle,

Narvaez, D. (2010). Moral Complexity: The Fatal Attraction of Truthiness and the Importance of Mature Moral Functioning. Perspectives on Psychological Science, 5(2), 163-181. doi: https://doi.org/10.1177/1745691610362351

Dalla, C. Deodato, S., Da Silva, M. \& Campos, E.. (2016). Elements and strategies for ethical decision-making in nursing. Texto \& Contexto. Enfermagem, 25(2), e4500014. Epub July 7, 2016. https://dx.doi.org/10.1590/010407072016004500014

Numminen, O. \& Leino-Kilpi, H. (2007). Nursing students ethical decision making a review of literature. Nurse education today, 27, 796-807.

Park, H.A., Cameron, M.E., Han, S.S., Ahn, S.H., O.H, H.S. \& Kim, K.U. (2003). Korean nursing students' ethical problems and ethical decision making. Nursing Ethics, 10(6), 638-653.doi: 10.1191/0969733003ne653oa

Park, E.J. (2012). An integrated ethical decision-making model for nurses. Nursing Ethics, 19(1), 1-5. doi: 10.1177/0969733011413491.

PLUS: The decision Making Process (s.f.). Retrieved from: http://www.burtbertram.com/teaching/ethics/Article_02-PLUS_DecisionMakingModel.pdf

Schmidt, L. (2008). Método de Interpretación y Análisis Holístico de Casos bioéticos. Acta Bioethica, 14(1), 39-46. Doi: http://dx.doi.org/10.4067/S1726569X2008000100005

Shapiro, J. \& Miller, R. (1994). How medical students think about ethical issues. Academic Medicine, 69(7), 591-593. Retrieved from: https://journals.lww.com/ academicmedicine/abstract/1994/07000/how_medical_students_think_about_ethical_issues.18.aspx

Sinclair, JM. (2013). New Zealand nursing students' experiences ofethical issues in clinical practice: A descriptivestudy. Master's tesis. Taradale: Eastern Institute of Technology New Zealand. Retrieved from: https://repository.digitalnz. org/system/uploads/record/attachment/661/new_zealand_nursing_students__experiences_of_ethical_issues_in_clinical_practice__a_descriptive_study.pdf

Souza, D. N. de \& Souza, F. N. de. (2014). Nursing Teaching Strategies by Encouraging Students' Questioning, Argumentation and Explanation. Revista da Escola de Enfermagem da UsP, 48(spe2), 155-163. Epub December 00, 2014.https://dx.doi.org/10.1590/S0080623420140000800023 
Schwartz, M. (2016). Ethical Decision-Making Theory: An Integrated Approach. Journal of Business Ethics, 139(4), 755-776. https://doi.org/10.1007/s10551-015-2886-8

Tsai, TC. \& Harasym, P. (2010). A medical ethical reasoning model and its contributions to medical education. Medical Education, 44(9), 864-873. Doi: https://doi. org/10.1111/j.1365-2923.2010.03722.x

Tanner, C. (2006). Thinking like a nurse: a research-based model of clinical judgment in nursing. Journal of Nursing Education, 45(6), 204-211. Retrieved from: https://pdfs. semanticscholar.org/717a/68a5a2eca79a95cle05bd595f9c10efdeafa.pdf?_ga=2.202142680.811302395.1549292946240716333.1540588243

The IDEA (2008). Ethical Decision-Making Framework. Toronto Central Community Care Access Centre Community Ethics Toolkit. Retrieved from: http:// www.trilliumhealthcentre.org/about/documents/TrilliumIDEA_EthicalDecisionMakingFramework.pdf

Thomasma, D. (2003). Theories of medical ethics: The philosophical structure. Military Ethics, Volume 1. Retrieved from: http://www.cs.amedd.army.mil/borden/Portlet. aspx?ID=106cb6b1-3327-47f3-a350-b2fb57beb928
Thompson, J. (1985). Bioethical decisión making for nurses. Norwalk, CT., Apleton-Century-Crofts.

Tong, R. \& Williams, N. (s.f.). Feminist Ethics. Stanford Encyclopedia of philosophy. Retrieved from: http:// plato.stanford.edu/entries/feminism-ethics/

Toren, O. \& Warner, N. (2010). Applying an ethical decision-making tool to a nurse management dilemma. Nursing Ethics, 17(3), 393-402. doi: https://doi.org/ $10.1177 / 0969733009355106$

Weizenbluth, J., Sokolowski, M. \& Gordon, M. (2008). The Power of Stories: Narrative Ethics in Long-Term Care. Annals of Long term care, 16(9): 26-29.

Weaver, K. (2007). Ethical Sensitivity: State of Knowledge and Needs for Further Research. Nursing Ethics, 14(2), 141-155. https://doi.org/10.1177/0969733007073694

Wueste, D. (2005). Philosophical yet user-friendly framework for ethical decision making in critical care nursing. Dimensions of Critical Care Nursing, 24(2), 70-79. doi: 10.1097/00003465-200503000-00006

Zhong, Chen-Bo (2011). The Ethical Dangers of Deliberative Decision Making Administrative Science Quarterly, 56, 1-25. https://doi.org/10.2189/asqu.2011.56.1.001 

